\title{
The Semantic Public Service Portal (S-PSP)
}

\author{
Nikolaos Loutas ${ }^{1,2}$, Deirdre Lee ${ }^{1}$, Fadi Maali ${ }^{1}$, Vassilios Peristeras ${ }^{3}$, \\ and Konstantinos Tarabanis ${ }^{2}$ \\ DERI, National University of Ireland Galway (NUIG), Galway, Ireland \\ \{nikos.loutas, deirdre. lee, fadi.maali\}@deri.org \\ Information Systems Lab, University of Macedonia, Thessaloniki, Greece \\ kat@uom.gr \\ European Commission, Directorate-General for Informatics, Interoperability Solutions for \\ European Public Administrations \\ vassilios.peristeras@ec. europa.eu
}

\begin{abstract}
One of government's responsibilities is the provision of public services to its citizens, for example, education, health, transportation, and social services. Additionally, with the explosion of the Internet in the past 20 years, many citizens have moved online as their main method of communication and learning. Therefore, a logical step for governments is to move the provision of public services online. However, public services have a complex structure and may span across multiple, disparate public agencies. Moreover, the legislation that governs a public service is usually difficult for a layman to understand. Despite this, governments have created online portals to enable citizens to find out about and utilise specific public services. While this is positive, most portals fail to engage citizens because they do not manage to hide the complexity of public services from users. Many also fail to address the specific needs of users, providing instead only information about the most general use-case. In this paper we present the Semantic Public Service Portal (S-PSP), which structures and stores detailed public-services semantically, so that they may be presented to citizens on-demand in a relevant, yet uncomplicated, manner. This ontologybased approach enables automated and logical decision-making to take place semantically in the application layer of the portal, while the user remains blissfully unaware of its complexities. An additional benefit of this approach is that the eligibility of a citizen for a particular public service may be identified early. The S-PSP provides a rich, structured and personalised public service description to the citizen, with which he/she can consume the public service as directed. In this paper, a use-case of the S-PSP in a rural community in Greece is described, demonstrating how its use can directly reduce the administrative burden on a citizen, in this case is a rural Small and Medium Enterprise (SME).
\end{abstract}

Keywords: eGovernment, public service, semantic, portal.

\section{Introduction}

Public service provision is an important duty of all government departments. Independent of what kind of service it is, every public service can be broken down into two distinct but complementary phases: the informative phase and the performative 
phase [1]. During the informative phase, the service provider provides information about the service to the citizen/ business ${ }^{1}$, while during the performative phase the citizen utilises the public service. The informative phase is essential for optimal service utilisation and public-administration efficiency, however it is often overlooked by governments. In order to effectively use a public service, citizens must identify which public services address their needs and find answers to their questions regarding these services, e.g. "am I eligible for this service", "what is the outcome of the service", "which public agency provides the service", etc. In this paper, we present a portal that facilitates the informative phase of public-service provision.

An additional factor of public services is that they often have complex structures, and may be specialized into a variety of service versions. For example a public service that is concerned with the issuing of a driving license, may have alternative versions if this is the first license of the applicant, if the applicant is over 60 , if the applicant wishes to drive lorries, etc. It is therefore not enough for citizens to identify a public service in general, but they must also go one step further and identify the specific service version for which they are eligible. The service versions refine the generic public service further and may be differentiated from one another according to:

i. the profile of the citizen that wishes to consume the service;

ii. the service inputs and outputs; and/or

iii. the service workflow.

However, traditional governmental portals still follow a one-size-fits-all approach. Thus the portal cannot react differently and tailor the offered public services to the needs and the profile of each individual citizen. Moreover, the citizen has to figure out on their own whether they are eligible for the service by reading lengthy public service descriptions (which very often include legal terminology). These are common problems in all existing national eGovernment portals. According to [2], the most typical problems of eGovernment portals can be grouped into the following categories:

- The user is unable to find the desired information or service.

- The user is unable to achieve his goal, even though the system supports it and he has started along the path to achieve it.

- The user is able to accomplish his goal, but not efficiently, e.g., easily and quickly.

In order to enhance the informative part of public service provision and improve existing governmental portals, this paper introduces the Semantic Public Service Portal (S-PSP), which aims:

- To inform citizens whether they are eligible for a specific public service;

- To personalize the public-service-related information according to the profile and the specific needs and wants of the citizen and identify the specific public service version;

\footnotetext{
${ }^{1}$ For the remainder of the paper we refer only to citizens for the sake of brevity, but citizens and businesses are implied.
} 
- $\quad$ To provide complete and well-structured information for the public service; and

- To allow citizens to invoke public services that are available online (if a service execution environment is in place).

The S-PSP was initially developed in the context of the SemanticGov project ${ }^{2}$, where it played the role of the national Member State portal [3]. It served as an entry point for the citizens to the public services offered by the SemanticGov platform. Two prototypes of the portal were installed at the Region of Central Macedonia in Greece and the City of Turin in Italy [4]. Currently, the portal is one of the three building blocks of the Rural Inclusion ${ }^{3}$ platform, as will be described in more detail in section 5. A running prototype of the portal is available at http://vmsgov03.deri.ie:8080/ruralinc/services?pilot=gr\&pageLanguage $=$ en

The remainder of the paper is structured as follows. Section 2 discusses related efforts. The S-PSP is presented in section 3, along with an overview of its architecture. The ontologies that the S-PSP used are discussed in section 4. An example of the S-PSP in use in the context of the Chios Chamber of Commerce is presented in section 5. Finally, our conclusions and future research directions are discussed in section 6 .

\section{Related Work}

Researchers have tried to solve parts of the problem that we described in the previous section, focusing mostly on facilitating service search and discovery. Fang et al. [5] support the selection of an optimal set of featured service-links. These links will then appear on the homepage of an eGovernment portal, thus helping users to locate services more easily by reducing the number of steps that they have to perform until the desired service is found. This is expected to improve the citizens' satisfaction and consequently increase the number of people using the portal. Therefore, a heuristic Web-mining algorithm called ServiceFinder is proposed, which aims to help citizens find the services that they are looking for in eGovernment portals. ServiceFinder uses three metrics to measure the quality of eGovernment service selection, which will then appear as links on the homepage of the portal. These are effectiveness (degree of easiness to locate the desired service), efficiency (probability to locate the desired service) and utilization (sum of easily located desired services). The metrics are calculated using patterns either extracted from the structure of the eGovernment portal or mined from a Web log. Although this approach may improve the service discovery by organizing better the available services within the portal, the process of finding a service in the portal is still based on a trial and error approach. This means that the user is still browsing the eGovernment portal in order to find the desired service. Another drawback of this approach as compared to ours is that it provides no information to the citizen with respect to his/her eligibility for the identified public service.

\footnotetext{
2 http://www.semantic-gov.org

3 http://www.rural-inclusion.eu
} 
Sacco [6] proposes a solution enabled by dynamic taxonomies, which support different facets that may be used by citizens. The facets that the system provides are: services, events of life, type of information, location, type of citizenship, person with special rights and person profile. The use of dynamic taxonomies makes this approach very flexible and fast. This is due to the fact that dynamic taxonomies adapt dynamically to the subset of the universe on which the user is focusing. The use of multiple facets enhances further the agility of the approach. Nonetheless, this work suffers from similar problems as other approaches that organize services in hierarchical category trees. The user may have to browse deep into the dynamic taxonomy and should also be aware of the way that public administration has decided to organize services (even if multiple facets are made available), which may differ from his/her perspective. Therefore, the cognitive effort of citizens is not reduced as much as expected. The eligibility question remains unanswered in this case as well.

Recently, Stollberg and Muth [7] proposed an approach for service customization which is based on simplified service variants that expose only the service features that are relevant to the profile of a specific user or to the characteristics of a specific usage scenario. Hence, the authors define $(i)$ the service variability metamodel, $(i i)$ the main actors and (iii) the lifecycle of the service customization process. They also provide a set of tools based on the metamodel to support the service variant creation. This work approaches the problem of service customization from a different perspective than the one implemented in our work. However, we too acknowledge the need to formalize the process, we thus use the Public Service Ontology described in section 4 to model public services and the SBVR standard [8] for formally expressing the business rules that lead to different service versions.

The OneStopGov project ${ }^{4}$ delivered a life-event portal that supports the active, citizen-centric approach [9]. The portal follows a structured dialogue approach, based on workflows that model life-events in order to personalise them to citizen profiles and facilitate their execution. Hence, the OneStopGov approach adapts the life-event to citizen's profile, which practically means that citizens with different profiles will most likely execute different versions of the same life-event. In the FIT project ${ }^{5}$, an adaptive eGovernment portal has been implemented [10]. The approach, which employs Semantic Web and Web 2.0 technologies, proposes a framework which captures the user's behaviour in the portal and adapts the portal accordingly. FIT's approach also recognizes the service version problem that we have described earlier and tries to overcome this by providing personalized eGovernment services. In order to achieve this, the FIT portal follows an iterative ontology-driven adaptation approach (monitor, analyze, plan, execute). The FIT portal also uses OWL ontologies to model users, events and adaptation rules.

The portal presented in this paper tries to fulfil similar objectives like the related efforts described so far. Nevertheless, some differences can be spotted both in terms of functionalities provided and in terms of the technologies used. For example, none of the related efforts decide on the eligibility of the citizen for a public service before

\footnotetext{
${ }^{4}$ www. onestopgov-project.org

${ }^{5}$ http: //www.fit-project.org/
} 
the execution of the service. This is a very strong asset of our approach, as the eligibility check at an early stage during the informative part of service provision can save the citizen a lot of time and money. It is interesting that the work of [6] bears some resemblance with ours in the way that services are modelled and organized, but what is different, apart from the use of ontologies versus taxonomies, is the fact that in our work services are described at a greater level of granularity e.g. the distinction between service type and service version. This difference is very important and due to this Sacco's work is not able to personalize services, but only provide generic info about them. Moreover, it does not answer the eligibility question.

\section{Semantic Public Service Portal (S-PSP)}

The Semantic Public Service Portal (S-PSP) provides information about available public-services, which a user may browse and search in a customisable and userfriendly manner. Through the use of the S-PSP, a user can quickly identify:

- which public service(s) are applicable to their individual use-case,

- whether they are eligible for these public service(s), and

- what is required from the user to complete these public service(s) for their individual use-case.

Fig. 1 shows the homepage of the S-PSP with the list of all currently available public services and the languages they are available in.

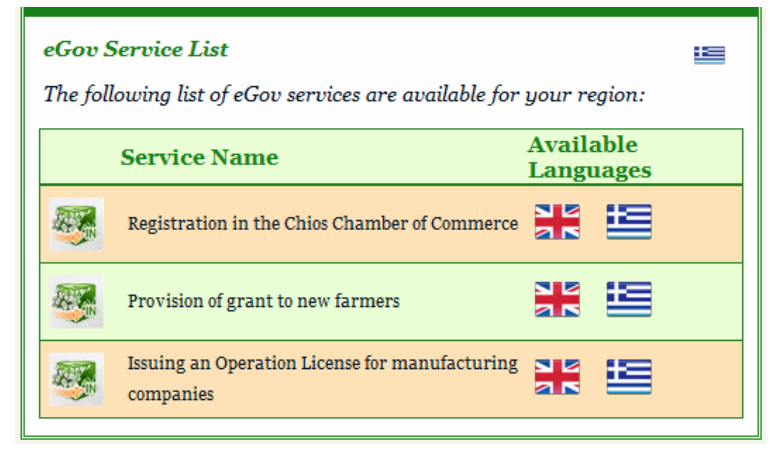

Fig. 1. The list of public services available in the S-PSP

Once a user selects the public service they are interested in, the dialogue page appears, as shown in Fig. 2. At this step, the user answers a series of questions, which will determine if a user is eligible for this service and what information they will need to provide/complete to utilise this service. Fig. 3 shows the customised information that this particular users requires to utilise this service, moving from a one-size-fits-all approach that is unrealistic in the case of public services. 


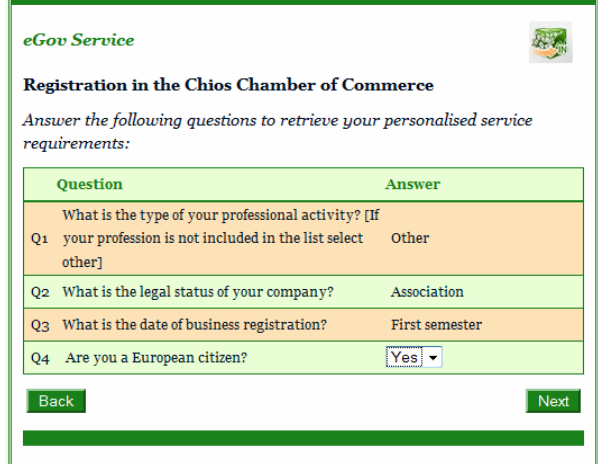

Fig. 2. The public-service dialogue to customise the public-service information

\begin{tabular}{|c|c|c|}
\hline \multicolumn{3}{|l|}{ Complete Document List } \\
\hline Name & Description & \\
\hline L ID or Passport & Copy of ID card or passport & More \\
\hline Application & $\begin{array}{l}\text { It is provided by Chios Chamber of } \\
\text { Commerce }\end{array}$ & More \\
\hline $\begin{array}{l}\text { Detachment of general } \\
\text { assembly and board of } \\
\text { directors proceedings }\end{array}$ & $\begin{array}{l}\text { It is produced by the Accountant of } \\
\text { the Company }\end{array}$ & More \\
\hline L $\begin{array}{l}\text { Business inception } \\
\text { certificate }\end{array}$ & $\begin{array}{l}\text { This document is provided by the } \\
\text { Tax Authority }\end{array}$ & More \\
\hline L Article of Incorporation & $\begin{array}{l}\text { This document is produced by the } \\
\text { Greek Court }\end{array}$ & More \\
\hline \multicolumn{3}{|c|}{ The following information is related to this service } \\
\hline You have to submit & Registration fee of 40 & \\
\hline You have to submit & Annual fee of 110 & \\
\hline
\end{tabular}

Fig. 3. The customised information required to utilise this public service

\subsection{S-PSP Architecture}

The S-PSP follows a three-tier architecture, as shown in Fig. 4, which comprises of:

- the User Interface Layer, which facilitates the interaction between the citizens and the portal, acting as an entry-point to the portal's functionalities.

- the Application Layer, which implements the functionalities provided to the citizens. This layer consists of two components:

$\circ$ the Service Tree Locator (STL) and

○ the Query Mechanism.

- the Semantic Repository Layer where all the semantic artefacts (ontologies) used by the portal are stored. 
While the user interacts directly with the S-PSP interface, the service provider collaborates with an ontology manager to define the public-service descriptions, which are then added to the Semantic Repository. This process of semantic publicservice description is currently in the process of being automated, so that the service provider may create the service description using a tool that will substitute the ontology manager.

\section{The User Interface Layer}

The User Interface (UI) Layer provides citizens with the means to interact with the portal. Its main functionality includes presenting the questions asked by the Query Mechanism to the citizens and collecting their answers. The answers are then returned to the Query Mechanism. It is important to clarify that all information that is made available through the UI, e.g. list items in dropdown lists, questions and possible answers etc., comes from the underlying ontologies stored in the Semantic Repository.

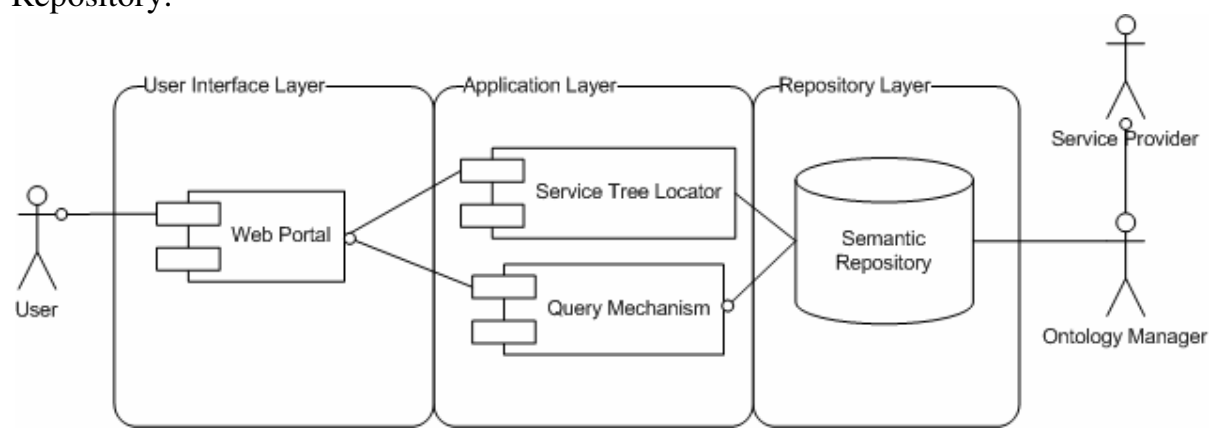

Fig. 4. S-PSP Architecture

\section{The Application Layer}

The Application Layer consists of the Service Tree Locator (STL) and the Query Mechanism components. The Service Tree Locator (STL) identifies the appropriate Service Tree Ontology (STO), which models the public service that addresses the user's requirements. Citizens can enter keywords in the portal's UI to describe the service that they are looking for. These keywords are sent to the STL, which then queries the semantic repository using SPARQL ${ }^{6}$ queries to find matching publicservice descriptions. The STL may contact $\mathrm{WordNet}^{7}$ in order to find synonyms and hypernyms/hyponyms for the keywords entered by the user, thus making the keyword search more effective. Finally, the resulting public services are returned to the citizens in order for them to select the appropriate one. SPARQL was chosen as the semantic query language, as it is a W3C Recommendation and has a large, active community.

The Query Mechanism (QM) is the core component of the S-PSP as it identifies the questions to include in the public-service dialogue, based on a user's previous

\footnotetext{
${ }^{6}$ http: / / www.w3 . org/TR/rdf-sparql-query/

${ }^{7}$ http: / / wordnet.princeton.edu/
} 
answers. The QM continually checks the user's eligibility for the public service and, if eligible, it stores the user's answers in order to determine the personalised information required to provide the service.

\section{The Repository Layer}

The Repository Layer contains the semantic repository component, which houses all of the ontologies of the S-PSP. These will be discussed in more detail in the next section.

\section{Semantic Description of Public Services}

The S-PSP structures and stores detailed public-services semantically. This ontologybased approach enables automated and logical decision-making to take place in the application layer of the portal, while the user remains unaware of its complexities. Adopting an ontology-based approach reaps the benefit of the flexibility of the RDF model. This, together with the highly abstract, conceptual nature of RDF triples, enable direct translation of fairly complex service scenarios into a machine-readable form. Inference over OWL ontologies helps achieve more concise and readable service descriptions. As a simple example, defining Schengencountry as a rdfs:subclassof EuropeanCountry class, means that any instance of SchengenCountry is also an instance of Europeancountry and eliminates the need for explicit listing of such a condition. However, in order to create semantic public service descriptions in this way requires a detailed analysis of the public description. This is usually carried out by an ontology engineer, in close conjunction with a civil servant who is extremely familiar with the intricacies of the public service. While this may be seen as a limitation of the approach, it results in a simple and effective tool from the citizen side. We are also in the process of creating a public-service description tool, which will replace the need for an ontology engineer to assist the civil servant in describing a public service semantically. The different kinds of ontologies that are utilised by the S-PSP are:

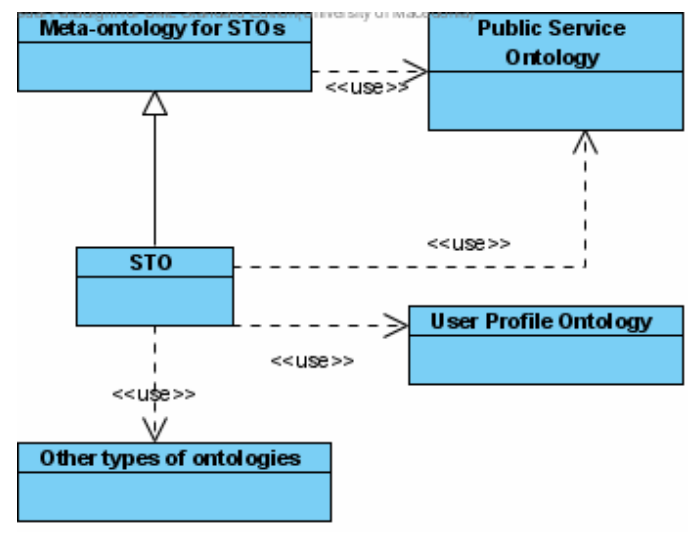

Fig. 5. Ontologies used by the S-PSP 
- $\quad$ Service Tree Ontology (STO)

- Public Service Ontology

- User Profile Ontology: models user preference and localization information.

- Other domain-specific Ontology: captures business information needed for service description.

We describe the details of the main ontologies used in the following sections.

\subsection{Service Tree Ontology}

A Service Tree Ontology (STO) formally defines the dialogue that would usually take place between a public-service provider and a citizen for a particular public service. STOs have a tree-like structure and are written in OWL. The dialogue starts from a generic public service, which is stepwise refined after every question/answer pair. In the case that the citizen is eligible for the specific public service, the dialogue leads to the public-service version that matches their profile and a detailed structured description of the public service version is made available. Otherwise the citizen is informed that they are not eligible for the specific public service. STOs contain the business rules from which the different service versions derive as well as the questions that will be asked to the citizen in order to collect information, which enables the portal to personalize the public service and decide on the eligibility of the citizen and on the matching service version. Moreover, the user interface of the portal is dynamically created based on information encoded in the STOs.

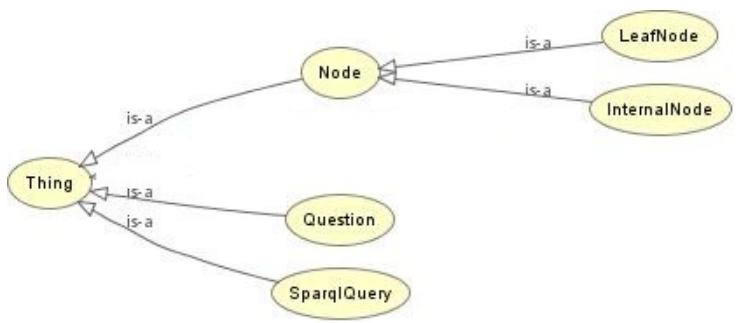

Fig. 6. The meta-ontology for STOs

In order to formalize the development of STOs, we have created a meta-ontology that defines the classes that comprise an STO. Each STO is then created as an instance of the meta-ontology. The meta-ontology contains classes that have been derived from the modelling of the aforementioned dialogue, as shown in Fig. 6.

- Node. Nodes of an STO represent different states of the dialogue. A node has the following attributes:

- The hasDescription attribute provides a brief description of the node, as to what the node represents in the STO.

- The hasEvidencePlaceholder attribute refers to the administrative documents, e.g. certificates, which relate to a specific service version. A Node may contain zero or more EvidencePlaceholders. 
- The containsPieceOfInformation attribute refers to other types of information related to a specific service version, e.g. the amount of a fee that has to be paid. A Node may contain zero or more PieceOfInformation.

The following three classes, i.e. InternalNode and LeafNode have been defined in the ontology as subclasses of Node. They thus inherit all its attributes.

- InternalNode. This class represents those nodes of the STO that have descendants. Apart from the attributes that they inherit from Node, InternalNodes have also:

- The hasChildNode attribute which indicates the descendants of the current node. There can be more than one descendants, which constitute specializations of their parent node, mainly by containing more information about the citizen's profile.

- The hasQuestion attribute which refers to a specific question asked to the citizen.

o The isRoot attribute which indicates whether the specific node is the initial node of the dialogue or not.

- LeafNode. This class represents those nodes of the STO that have no descendants. LeafNodes indicate the termination of the dialogue, whether successful or not. Apart from the attributes that they inherit from Node, LeadNodes have also the isNotEligible attribute which if true indicates that the citizen is not allowed to use the specific public service.

- Question. This class represents the questions that the portal poses to the citizen. It has two attributes:

o hasData models the question itself, e.g. "What is your marital status?"

- hasAnswer models the possible answers, e.g. in the previous question "married, single, divorced, widow".

- SparqlQuery. This class represents formally a business rule of a specific public service. The business rule is expressed as a SPARQL query which is stored in the hasData attribute.

\subsection{Public Service Ontology}

The S-PSP capitalizes on the GEA Public Service Model implementation in OWL (Public Service Ontology) [11]. The Public Service Ontology, which is depicted in Fig. 7, is used for representing public service related information by creating instances of the various classes that comprise the description of the public service. A brief description of the Public Service Ontology's classes follows. Societal Entities (e.g. citizen, business) have Needs related to specific Goals. A Societal Entity requests a Public Administration (PA) Service to serve its Goals. PA Services are categorized in several Domains (e.g. Health, Transportation). Each Domain object is divided into several SubDomain objects (e.g. Domain Transportation has SubDomains Ground Transportation, Air Transportation and Water Transportation). There are several types of Social Entities (e.g. legal entity, physical person). There are two categories of Governance Entities participating in service provision: Political Entities and Public Administration Entities. Based on the role which $P A$ Entities can acquire during the service execution phase, we identify four Roles: 


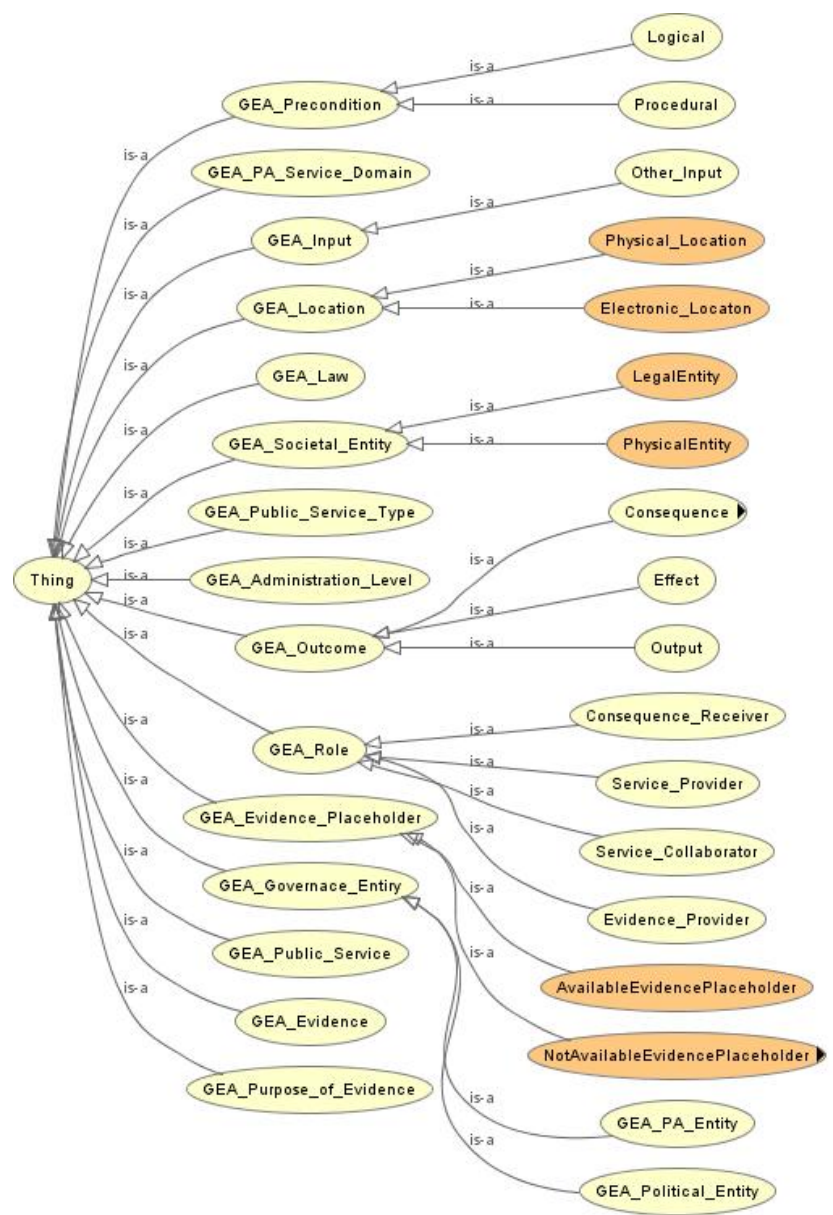

Fig. 7. The Public Service Ontology

- Service Provider is the PA Entity that provides the service to the Societal Entities (clients). The PA Entities belong to an Administrative Level (e.g. municipality, regional).

- Evidence Provider is the PA Entity that provides necessary Evidence to the Service Provider in order to execute the PA Service.

- Consequence Receiver is the PA Entity that should be informed about a PA Service execution.

- Service Collaborator is the PA Entity that participates in the provision of a public service (but is not the service provider).

Political Entities define PA Services which are governed by Preconditions usually specified in Legal Acts - Laws. Preconditions set the general framework in which the service should be performed and the underlying business rules that should be fulfilled 
for the successful execution of the PA Service. Preconditions can be formally expressed as a set of clauses. Preconditions are validated by Piece of Evidence serving a Purpose. As Evidence is primarily pure information, it is stored in Evidence Placeholders, thus the Evidence Placeholder contains Pieces of Evidences. PA Service use specific types of Evidence Placeholders as Input. The Outcome refers to the different types of results a PA Service may have. GEA defines three types of Outcome:

- Output, which is the documented decision of the Service Provider regarding the service asked by a Societal Entity. This is currently embedded and reaches the client in the form of an Evidence Placeholder.

- Effect, which is the change in the state of the real world (e.g. transfer money to an account) caused by the execution of a service. In cases where administration refuses the provision of a service, there is no Effect.

- Consequence, which is information about the executed PA Service that needs to be forwarded to interested parties.

\subsection{Other Ontologies}

In addition to the STOs, the meta-ontology for STOs, and the Public service ontology, the following OWL ontologies are also used by the S-PSP:

- Ontologies that model the profile of businesses and citizens, for example the brand name, type, or legal status.

- Ontologies that contain public service related information, such as the administrative documents that are required as input for the different versions of the public service (modelled as instances of the EvidencePlaceholder class of the Public Service Ontology).

- Ontologies that include listings of countries, nationalities, and business types.

\subsection{Query Mechanism's (QM) Usage of an STO}

The QM, as discussed in section 3.1, is the core component of the S-PSP, as it identifies the questions to include in the public-service dialogue, by traversing the corresponding public-service STO. During the traversal of the STO, the public service that the citizen has selected is being personalized according to their answers. This is achieved by resolving the generic service type into the appropriate service version. It is important to note that at each stage of the traversal, the next step option is unique. This means there is no case where the same citizen could follow two different paths in the same STO. If the current node is an InternalNode then the QM has to verify the conditions of all its descendants, which are expressed as SPARQL queries. Therefore, the QM takes the appropriate question from the STO and forwards it to the UI so that the question can be displayed to the citizen. In case the current node is a LeafNode, i.e. it has no descendants, then the end of the structured conversation has been 
reached. At this point the portal has collected all the necessary information for identifying the specific public service version that matches the citizen's profile and for deciding on their eligibility. In case the citizen is not eligible for one of the service versions that are modelled in the STO (isNotEligible is set to true), then the QM terminates its execution and returns a notification message, for example, 'You are not eligible for this service because you are under 18 years old'.

QM Traversal Process in Pseudocode:

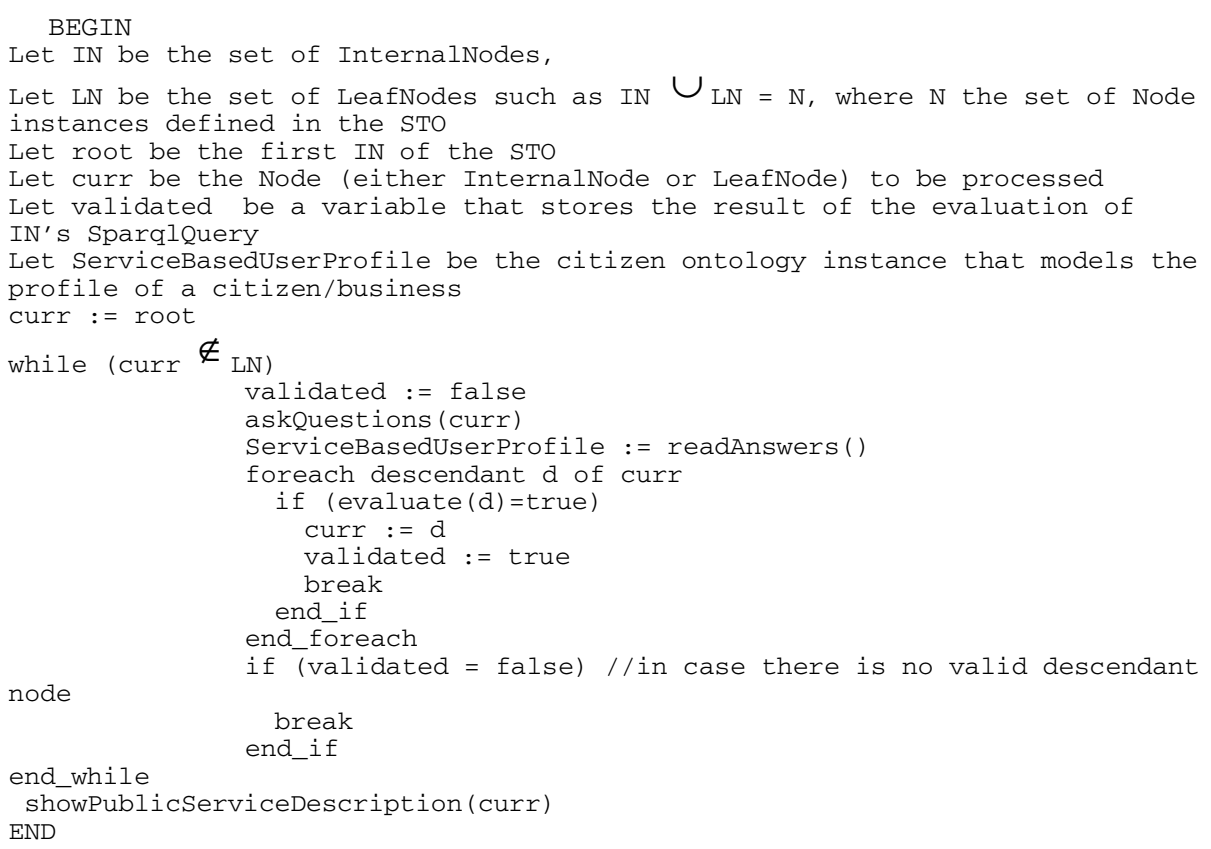

\section{S-PSP In-Use: Rural Inclusion Trial-Site Chios}

The S-PSP is currently being utilised by Rural Inclusion ${ }^{8}$, an EC project funded under the Competitiveness and Innovative Framework Programme. Rural Inclusion aims at adopting a state-of-art infrastructure that will facilitate the offering of innovative services by public administration in rural areas. The S-PSP is one of three components that make up the Rural Inclusion Platform, which is being rolled out across five European trial-sites in France, Martinique, Greece, Latvia and Spain.

The Chios Chamber of Commerce is one of the trial-partners. It is supervised by the Greek Ministry of Development and serves as a consultant of a wide range of business-related matters for the Greek island of Chios. Public services that the chamber provides and that are presented in the Rural Inclusion platform include:

${ }^{8}$ http: / /www.rural-inclusion.eu 
- Registration of a new business in Chios

- Provision of grants to new farmers

- Issuing of operation licenses for manufacturing companies

In this section, we will present how the 'registration of a new business in Chios' public service is modelled using the S-PSP ontologies. Although this service sounds straight-forward, there are actually more than 20 different versions which stem from variations in the legal status of the enterprise/entrepreneur, on the type of their activity, and on their nationality. Initially, a documentation exercise was carried out with the service provider, in order to gather all the details with regards to the provision of the specific service. Hence, the business rules that are associated with this public service were identified and the activity diagram of the structured dialogue was designed. This was then encoded into an OWL ontology by the ontology manager.

For example, one of the business rules of the 'registration of a new business' public service expressed in structured English (following SBVR [8]) reads "It is obligatory that each SA company with equity capital less than 64,000 Euros pays an annual fee of 160 Euros." In order to collect the information that validates this rule, a question was created asking the equity capital of the SA company, i.e. "Please provide the equity capital of your company". In the STO developed for this public service, this question was then attached to one of the InternalNodes. This specific InternalNode has three descendants. One of them will correspond to the case of an SA company with capital less than 64,000 Euros. This will be expressed by a SPARQL expression linked to this specific node, as shown below.

\section{The InternalNode instance}

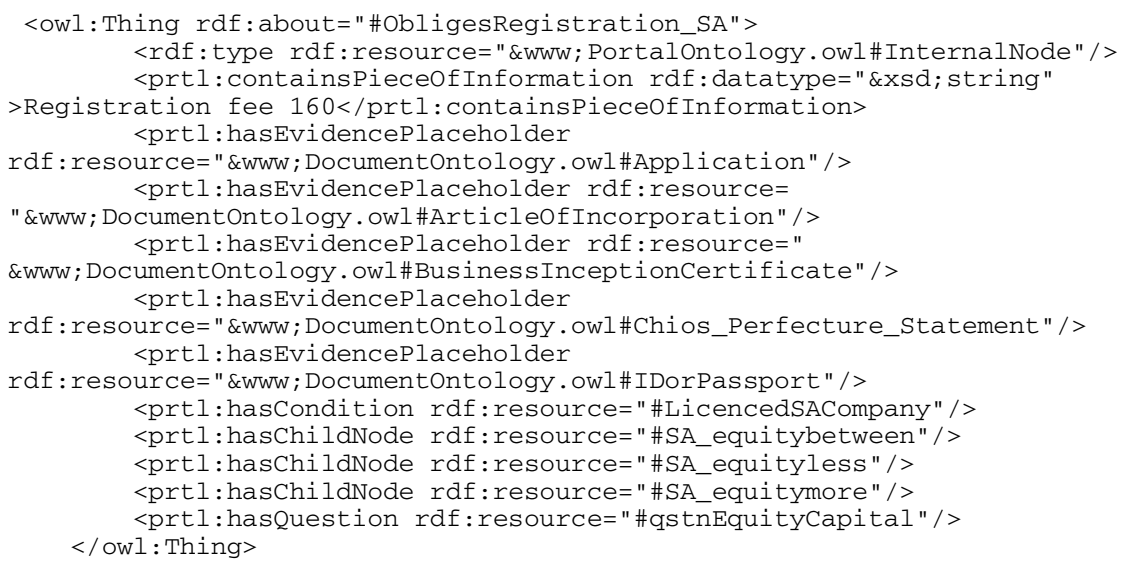

\section{The Question instance}

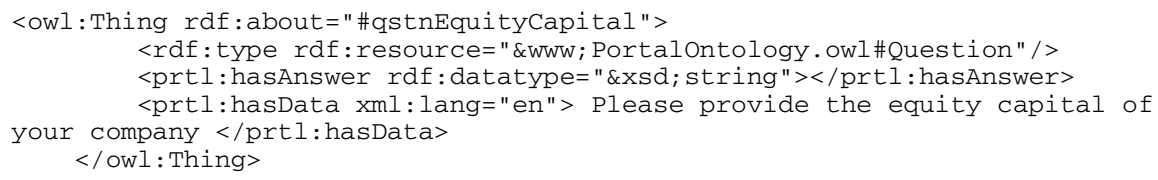




\section{The SparqlQuery instance}

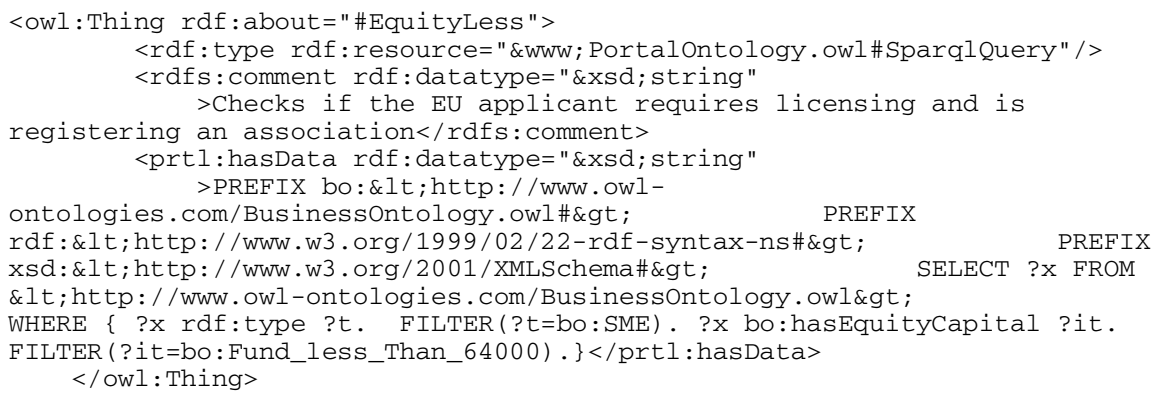

\section{Evaluation}

The evaluation of the S-PSP is ongoing as part of the Rural Inclusion Project. As stated previously, the S-PSP is one of three components that make up the Rural Inclusion Platform, which is being rolled out across five European public-sector trialsites in France, Martinique, Greece, Latvia and Spain. Initial results are positive, with the main constructive criticism focusing on improving the intuitive integration of the S-PSP into the actual trial-partner sites, and where they currently provide the actual public services. A complete evaluation will be published at a future date.

\section{Conclusion}

This paper presents an ontology-based, public-services portal, the S-PSP, which facilitates the informative phase of public service provision. It checks the eligibility of the citizens for a specific public service before the actual execution of the service, thus saving them time, effort and money. Also the public service related information is personalised according to the profile and the specific needs and wants of the citizen and the specific public service version required is identified, thus providing targeted, tailored and comprehensive information. The S-PSP's architecture is modular and as such it is easily extendable. This has been shown with the Rural Inclusion Platform, where the S-PSP has been integrated with other components for a specific solution. The S-PSP is also decoupled from the public-service execution environment that may be available in different technologies and communicates with it using Web Services. The main advantage of this portal is its use of semantics to describe all aspects of public-services, resulting in reusable, extensible public-service data. New publicservices may be added to the portal through the creation of a new STO.

Acknowledgments. The work presented in this paper has been funded in part by Science Foundation Ireland under Grant No. SFI/08/CE/I1380 (Lion-2) and the European Union under Grant No. CIP-ICT PSP-2008-2/238900 (Rural Inclusion). The authors would like to thank all the Rural Inclusion project partners for the creative discussions and ideas. 


\section{References}

1. Peristeras, V., Tarabanis, K.: The Governance Architecture Framework and Models. In: Advances in Government Enterprise Architecture, IGI Global Information Science Referencece (2008)

2. Thomas, S., Schmidt, K.U.: D4: Identification of typical problems in eGovernment portals, in Technical Report, FIT Consortium (2006)

3. Loutas, N., Peristeras, V., Tarabanis, K.: Providing Public Services to Citizens at the National and Pan-European level using Semantic Web Technologies. In: 6th Eastern European eGov Days, Prague, Czech Republic (2008)

4. Loutas, N., et al.: A Semantically Enabled Portal for Facilitating the Public Service Provision. In: Semantic Technologies for E-Government, pp. 287-314. Springer, Berlin (2010)

5. Fang, X., Liu Sheng, O.R., Chau, M.: ServiceFinder: A Method Towards Enhancing Service Portals. ACM Transactions on Information Systems (TOIS) 25(4) (2007)

6. Sacco, G.M.: Interactive Exploration and Discovery of eGovernment Services. In: 8th Annual International Conference on Digital Government Research: Bridging Disciplines \& Domains, Philidelphia, US (2007)

7. Stollberg, M., Muth, M.: Service Customization by Variability Modeling. In: Service Customization by Variability Modeling, 5th International Workshop on Engineering Service-Oriented Applications (WESOA 2009) co-located with the ICSOC-ServiceWave, Vienna, Austria (2009)

8. OMG, Semantics of Business Vocabulary and Business Rules (SBVR) V1.0 (2008), http: / /www. omg.org/spec/SBVR/1.0/

9. Tambouris, E., Vintar, M., Tarabanis, K.: A life-event oriented framework and platform for one-stop government. In: 4th Eastern European eGov Days, Prague, Czech Republic (2006)

10. Schmidt, K.U., et al.: Personalization in e-Government: An Approach that combines Semantics and Web 2.0, in: Semantic Technologies for E-Government. In: Vitvar, T., Peristeras, V., Tarabanis, K. (eds.) Semantic Technologies for E-Government, pp. 261285. Springer, Berlin (2010)

11. Peristeras, V., et al.: Ontology-Based Search for eGovernment Services Using Citizen Profile Information. Journal of Web Engineering 8(3), 245-267 (2009) 